Recepción: 04 / 09 / 2018

Aceptación: 23 / 11 / 2018

Publicación: 03 / 12 / 2018

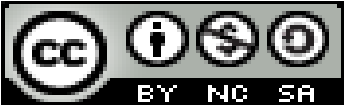

Ciencias de la salud

Artículo científico

\title{
Análisis del servicio de cirugía buco maxilofacial en la ciudad de Manta
}

Analysis of the oral maxillofacial surgery service in the city of

\section{Manta}

Análise do serviço de cirurgia buco maxilo-facial na cidade de Manta

\author{
Sandy S. García-Arcentales ${ }^{\mathrm{I}}$ \\ sandyst_gatciaar@gmail.com \\ María V. Andrade-Valencia II \\ ma.victoria_andradev@gmail.com
}

Correspondencia: sandyst_gatciaar@gmail.com

\footnotetext{
I Odontóloga rural, Ministerio de Salud Pública del Ecuador, Manta, Ecuador.

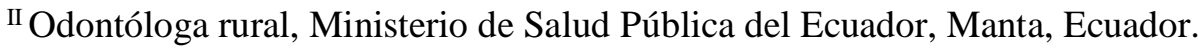




\section{Resumen}

Para este trabajo, se realizó un estudio observacional analítico transversal de 100 pacientes atendidos en el Servicio de Cirugía Maxilofacial de una clínica odontológica particular en Manta, en el período de enero 2017 a diciembre 2017, con la finalidad de evaluar sus resultados en intervenciones complejas. Predominó el sexo masculino con el 61,3\% y dicha cirugía se realizó con mayor frecuencia entre los 28 y 37 años de edad. Las intervenciones más frecuentes fueron la reducción de fracturas orbitocigomáticas y nasales, cirugía sinusal y rinoplasia correctora, y los procederes quirúrgico y anestésico fueron satisfactorios, con escasas complicaciones posoperatorias no inherentes a estos. El proceder proporcionó un alto grado de satisfacción al paciente.

Palabras clave: Salud oral; cirugía bucal; procedimientos quirúrgicos orales; rinoplastia; senos paranasales; fracturas cigomaticas; cirugía maxilofacial.

\section{Abstract}

For this work, an analytical observational cross-sectional study of 100 patients treated in the Maxillofacial Surgery Service of a particular dental clinic in Manta was carried out, through the ambulatory major surgery system, in the period from January 2017 to December 2017, for the purpose of to evaluate their results in complex interventions. Male sex predominated with $61.3 \%$ and said surgery was performed more frequently between 28 and 37 years of age. The most frequent interventions were the reduction of orbitozygomatic and nasal fractures, sinus surgery and rhinoplasty, and the surgical and anesthetic procedures were satisfactory, with few postoperative complications not inherent in these. The procedure provided a high degree of patient satisfaction.

Keywords: Oral health; oral surgery; oral surgical procedures; Rhinoplasty; sinuses; zygomatic fractures; maxillofacial surgery.

\section{Resumo}

Para este trabalho, um estudo de observação em corte transversal de 100 pacientes tratados com a cirurgia maxilo-facial de uma clínica dentária privada em Manta, pelo sistema de cirurgia ambulatória, no período de janeiro de 2017 para dez 2017 foi realizado com o objectivo avaliar seus resultados em intervenções complexas. O sexo masculino predominou com $61,3 \%$ e a 
cirurgia foi realizada com maior frequência entre 28 e 37 anos de idade. As intervenções mais frequentes foram reduzidos fraturas orbitozigomática e nasal, cirurgia sinusal e rinoplasia revisor, e procederes cirúrgicos e anestésicos foram satisfatórios, com poucas razões não relacionadas com estas complicações pós-operatórias. O procedimento proporcionou um alto grau de satisfação do paciente.

Palavras chave: Saúde bucal; cirurgia de boca; procedimentos cirúrgicos orais; rinoplastia; seios; fraturas zigomáticas; cirurgia maxilofacial.

\section{Introducción}

Las técnicas quirúrgicas han tenido gran avance desde el siglo XX, cada vez menos cruentas, y en el terreno de la Anestesiología, para brindar atención anestésica adaptada a las necesidades quirúrgicas. ${ }^{1}$ Esta modificación en la "forma" en que se practicaba la cirugía pudo ocurrir solo cuando el pronóstico y los resultados posquirúrgicos fueron similares, como mínimo, a los logrados dentro de los hospitales. ${ }^{2}$

Esta modalidad no es nueva, de hecho se reconoce como la forma más antigua de la práctica quirúrgica, ${ }^{3}$ sólo que ahora, ante la disyuntiva de los altos costos de la atención médica y la prestación de este servicio a la población, se volvió la vista a este método, para tratar de encontrar con él la solución a la problemática de atención quirúrgica de calidad. ${ }^{4}$ Ecuador, inmerso en importantes planes y metas de salud, no puede permanecer ajeno a este paso que aúna la atención primaria y secundaria con el objetivo de permitir una asistencia médica ininterrumpida al paciente y disminuir los efectos emocionales en el seno familiar.

Nuestra especialidad, por las características de los pacientes y sus enfermedades, ha encontrado en este proceder las bases necesarias para resolver con agilidad un significativo número de problemas de salud, que por su complejidad tenían una solución más prolongada anteriormente. Es aplicable hasta alrededor del $75 \%$ de los procedimientos de cabeza y cuello, donde se incluyen los traumatismos faciales, técnicas de extirpación y reconstrucción de tejidos, etc.3,5

El presente trabajo tiene como objetivo evaluar los resultados de esta modalidad en intervenciones complejas de III y IV nivel de Vélez6 y demostrar sus ventajas a través de la 
experiencia del servicio, en un intento por aportar datos que contribuyan a reforzar las bases científicas de quienes aseguren un buen servicio en la atención de la cirugía buco maxilofacial.

\section{Métodos}

Se realizó un estudio observacional analítico transversal en el Servicio de Cirugía Maxilofacial de una clínica odontológica particular en Manta, durante el período de enero 2017 a diciembre 2017. Se seleccionaron 100 pacientes según criterios establecidos ${ }^{3,6}$ de voluntariedad, estado físico I y II según ASA, medio social adecuado, posibilidad de seguimiento médico estrecho e intervención incluida en los niveles III y IV de Vélez, modificados por la especialidad según programas y centros de cirugía mayor ambulatoria. ${ }^{2,10}$

Los pacientes ingresaron el día del acto quirúrgico; una vez efectuado el ingreso, fueron trasladados a la unidad de observación hasta su egreso antes de las 24 h, seguidos por consulta externa en coordinación con el médico de familia. Se determinaron las intervenciones quirúrgicas, métodos anestésicos, complicaciones posoperatorias, costos hospitalarios y grado de satisfacción del paciente.

\section{Resultados}

La información se recolectó en una encuesta con variables demográficas y específicas. Se procesó en una microcomputadora IBM-compatible mediante el paquete de programas estadísticos MICROSTAT, realizándose estadística descriptiva e inferencial. Los resultados se reflejaron en tablas El grupo de edades donde más se empleó el sistema ambulatorio fue el comprendido entre los 28 y 37 años, lo que representó el 47,5 \% de los operados; al sexo masculino correspondió el $61,3 \%$.

La reducción de fracturas orbitocigomáticas fue la intervención más frecuente, con el 24,2\% (tabla 1) seguida por la reducción de fracturas nasales $(20,0 \%)$ y la antrostomía anterior y cierre de comunicación bucosinusal (C B-S) con 15,8 \%. En cuanto al tipo de anestesia utilizada, la general endotraqueal (GET) fue la más frecuentemente empleada $(60,0 \%)$ (tabla 2). 
Tabla 1. Frecuencia de las intervenciones quirúrgicas realizadas por niveles

\begin{tabular}{lll}
\hline Intervenciones quirúrgicas & No. & \% \\
\hline a) Nivel III & 29 & 24,2 \\
Reducción de fracturas orbitocigomáticas & 24 & 20,0 \\
Reducción de fracturas nasales & 19 & 15,8 \\
Antrostomía anterior y cierre de C B-S & 17 & 14,2 \\
Rinoplastia & 7 & 5,8 \\
Reducción de fractura mandibular & 7 & 5,8 \\
Submaxilectomía & 5 & 4,1 \\
Parotidectomía subtotal & 5 & 4,1 \\
Exéresis y plastia de carcinoma epidermoide labial & 3 & 2,4 \\
Reducción de fractura Lefort I & & \\
b) Nivel IV & 2 & 1,6 \\
Corrección de prognatismo mandibular & 2 & 1,6 \\
Corrección de prognatismo dentoalveolar maxilar & $\mathbf{1 2 0}$ & $\mathbf{1 0 0 , 0}$ \\
\hline Total & &
\end{tabular}

Tabla 2. Tipo de anestesia empleada

\begin{tabular}{lll}
\hline Tipo de anestesia & No. & \% \\
\hline General endotraqueal (GET) & 62 & 50,0 \\
Local y sedación (L/S) & 39 & 34,2 \\
General endovenosa (GEV) & 19 & 15,8 \\
\hline
\end{tabular}

$\mathrm{P}=1,677 \mathrm{E}-06$.

Se presentaron 8 complicaciones en el tiempo analizado para el 6,4\%, y de ellas, la sepsis de la herida $(2,4 \%)$ y el hematoma $(1,6 \%)$ fueron las más frecuentes (tabla 3$)$. 
Tabla 3. Complicaciones posoperatorias presentadas

\begin{tabular}{lll}
\hline Tipo de complicación & No. & $\mathbf{\%}$ \\
\hline Sepsis de la herida & 3 & 2,4 \\
Hematoma & 2 & 1,6 \\
Náuseas y vómitos no persistentes & 1 & 0,8 \\
Hemorragia & 1 & 0,8 \\
Dehiscencia de la herida & 1 & 0,8 \\
Total & $\mathbf{8}$ & $\mathbf{6 , 4}$ \\
\hline
\end{tabular}

La tabla 4 refleja los resultados de la encuesta de satisfacción practicada al alta definitiva, donde el $95 \%$ de los pacientes vertió una opinión favorable.

Tabla 4. Opinión sobre el método y el seguimiento

\begin{tabular}{lllllll}
\hline & \multicolumn{2}{c}{ Bueno } & \multicolumn{2}{c}{ Regular } & \multicolumn{2}{c}{ Malo } \\
& No. & $\%$ & No. & $\%$ & No. & $\%$ \\
\hline Opinión del método & 114 & 95,1 & 6 & 5,0 & 1 & 0,8 \\
Opinión del seguimiento & 113 & 94,2 & 5 & 4,1 & 1 & 0,8 \\
\hline
\end{tabular}

$\mathrm{P}=4,179 \mathrm{E}-63$

\section{Discusión}

Los resultados de nuestra investigación en relación con sexo y décadas más afectadas coinciden con otros autores, ${ }^{2,6,8,9}$ lo que está muy relacionado con las entidades tratadas. Se destaca en este aspecto que la edad no fue un factor excluyente como elemento único, sino la combinación de factores personales de salud anterior. Las intervenciones más frecuentemente realizadas coinciden con nuestro estudio, se aprecia la incorporación de entidades de tratamiento más complejo, según tendencias actuales, que abogan por el incremento de la diversidad y complejidad de las intervenciones realizadas ambulatoriamente. Se destaca la importancia de minimizar el tiempo quirúrgico y por inferencia la morbilidad, controlando factores como: estandarización de la instrumentación y secuencia quirúrgica, cuidadosa planificación preoperatoria, mínima manipulación de los tejidos, hemostasia perfecta, etc. 
Por la envergadura de las operaciones y el mayor tiempo quirúrgico fue necesario la aplicación de la anestesia general endotraqueal (GET) en un mayor número de pacientes. Para su éxito es importante la utilización de fármacos con duración breve, una semivida de eliminación rápida y baja incidencia de efectos secundarios como náuseas, vómitos y somnolencia. ${ }^{13} \mathrm{La}$ aparición de nuevos fármacos como el Midazolan, Propofol, Sevoflurane, Mivacurium, etc., hacen de esta la técnica de elección. ${ }^{12,13}$

Solo se presentaron complicaciones en 8 casos, para el 6,4\%, estos valores que coinciden con los parámetros de la Organización Mundial de la Salud (OMS), la cual plantea que el rango de complicaciones ha de encontrarse entre el 2,5 y el $12 \%$; además, consideramos que este aspecto se vio favorecido por el cuidado que se tuvo en cuanto a la asepsia, antisepsia, hemostasia y técnicas quirúrgicas; en la literatura revisada ${ }^{2,3,6-11}$ los porcentajes de esta complicación son similares a los nuestros.

El porcentaje de aceptación de este método a escala mundial se encuentra por encima del $89 \%$ en la literatura médica revisada, independientemente de la afección tratada; ${ }^{2,3,6}$ este hecho se repite en todos los estudios, que incluyen grupos poblaciones de múltiples países con variados grados de desarrollo socioeconómico y diferentes culturas. En nuestro trabajo el $95 \%$ lo consideró bueno acorde con los aspectos encuestados.

\section{Referencias Bibliográficas}

1. White PF, Smith I. Ambulatory anaesthesia: past, present and future. Int Anesth Clin 1996;32:1-16.

2. Dann JJ. Outpatient oral and maxillofacial surgery: Transition to a surgicenter setting and outcomes of the first 200 cases. J Oral Maxillofac Surg 1998; 56 (5):572-7.

3. Davis JE. Cirugía mayor ambulatoria. Clin Quirurg Norteamer 1987;67(4):685,749.

4. Duffy SQ, Farley DE. Patterns of decline among inpatient procedures. Public Health Rep 1995;110(5):682-3. 
5. Paredes Díaz JC, Hernández Pérez R, Castañeda González H, Rodríguez Otero AR. Cirugía mayor ambulatoria del paciente maxilofacial. Actualización. I Parte. Rev Cubana Estomatol 1991;28(2):106-11.

6. Paredes Díaz JC, Hernández Pérez R, Fernández Lastres D. Cirugía mayor ambulatoria del paciente maxilofacial. II Parte. Rev Cubana Estomatol 1991;28(2):112-7.

7. Haug RH. Selecting the appropiate setting for management of maxillofacial trauma. J Oral Maxillofac Surg 1999;57(8):983-9.

8. Martínez Subias J. Cirugía ambulatoria ORL. Acta Otorrinolaring Esp 1998;49(4):301-5.

9. Haug RH. Management of maxillofacial trauma in the ambulatory facility. J Oral Maxillofac Surg 1997;55(8):31-4.

10. Steckler RM. Outpatient parotidectomy. Am J Surg 1991;162:303-5.

11. Lupori JP, Van Sickels JE, Holmgreen WC. Outpatient orthognatic surgery: Review of 205 cases. J Oral Maxillofac Surg 1997;55:558-61.

12. Hutchings P. Advances in anesthesia. Some recent developments in techniques for stay surgery. Anaesth 1997;50(5):397-9.

13. Twersky R. The pharmacology of anesthetics used for ambulatory surgery. Anesth Analg 1995;81(1):101-12.

14. Pérez Garriguez T. Cirugía ambulatoria en ORL en un hospital de distrito. Acta Otorrinolaring Esp 1998;49(4):301-5.

15. Wong ME. Maxillofacial trauma treatment decisions based on outcome studies, host factors, and cost of care. J Oral Maxillofac Surg 1997;55(8):4-6.

16. Rhodes RS. Ambulatory surgery and the societal cost of surgery. Surg 1994;116(5):938-41.

17. Sandler NA, Braun TN. Economic analysis and its application to oral and maxillofacial surgery. J Oral Maxillofac Surg 1996;54(5):622-6. 